\title{
Current directions in behavioral medicine research on genetic testing for disease susceptibility: introduction to the special section
}

\author{
Kerry A. Sherman ${ }^{1,3} \cdot$ Linda D. Cameron ${ }^{2}$
}

Published online: September 8, 2015

(c) Springer Science+Business Media New York 2015

\begin{abstract}
The aim of this special section is to showcase research contributing to our understanding of factors influencing decisions to undergo genetic testing and the impact of the genetic testing process on health-related behaviors of tested individuals. The first two articles report studies investigating factors associated with interest in genetic testing and acceptance of test results (Sherman et al. in J Behav Med doi:10.1007/s10865-015-9630-9, 2015; Taber et al. in J Behav Med doi:10.1007/s10865015-9642-5, 2015b). The next two papers address the unique contribution of genetic risk information to understanding risk beyond genetic counseling alone (Heiniger et al. in J Behav Med doi10.1007/s10865-015-9632-7, 2015; Taber et al. in J Behav Med doi:10.1007/s10865015-9648-z, 2015a). The final three articles investigate the effects of genetic risk information on beliefs about disease control and prevention (Aspinwall et al. in J Behav Med doi:10.1007/s10865-015-9631-8, 2015; Kelly et al. in $\mathbf{J}$ Behav Med doi10.1007/s10865-014-9613-2, 2014; Myers et al. in J Behav Med doi:10.1007/s10865-015-9626-5, 2015). Collectively, the special section of papers highlights the diverse ways in which behavioural medicine contributes to our understanding of genetic testing for disease risk, and points to the value of further research to better understand ways in which individuals perceive, interpret and respond to genetic risk information.
\end{abstract}

Kerry A. Sherman

Kerry.sherman@mq.edu.au

1 Department of Psychology, Centre for Emotional Health, Macquarie University, Sydney, NSW 2109, Australia

2 Westmead Breast Cancer Institute, Macquarie University, Sydney, Australia

3 University of California, Merced, CA, USA
Keywords Genetic testing - Genetic risk - Psychosocial · Disease control · Preventive behaviour · Risk communication

This special section highlights the diverse ways in which behavioral medicine contributes to our understanding of genetic testing for disease risk. Since the mapping of the human genome in 2003, advances in genomic medicine have led to rapid and dramatic changes in the provision of healthcare (Patenaude, 2005). With information about genetic disease risk, health care providers can better personalize and modify treatment and support services to improve the health and well-being of their clients. There are currently more than 2000 known links between specific genetic mutations (faults) and disease risk across many conditions, ranging from cancers, diabetes, and heart disease through to multiple sclerosis, obesity, and Alzheimer's disease (National Institutes of Health, 2013). Singlegene tests to determine an individual's risk for a specific condition are currently available for those conditions where this link has been identified, with the number of available tests expanding at a rapid rate (National Institutes of Health, 2013). In addition, use of genomic sequencing methods that provide information about genetic risk for multiple health conditions is increasing rapidly (Beachy et al., 2015).

Initially, genetic testing services were solely the domain of clinical settings in which individuals received expert advice on the use of genetic testing, interpretation of results, and risk management (Williams \& Javitt, 2008). Over recent years, however, the development of cheaper, faster testing methods has led to the availability of genetic testing through direct-to-consumer online testing services (Caulfield et al., 2010; National Health and Medical 
Research Council, 2012; Ostergren et al., 2015). These advances in genetic testing, increasingly referred to as genomic testing, thus provide new opportunities for disease prediction, diagnosis, and treatment. Yet they also create a demand for associated shifts in health care and support services to help individuals comprehend their genetic risk status and make informed decisions throughout the course of the genomic testing, disease risk management, and treatment process. Behavioral medicine research provides critical evidence that can guide the development of these health care delivery and support services.

Key features of genomic tests driving this need for behavioral medicine research and interventions include the complexity and ambiguity of the test results and their implications. Genetic testing typically entails a saliva or blood test that identifies mutations, or changes, in chromosomes, genes, or proteins (Barlow-Stewart \& Parasivam, 2007; National Institutes of Health, 2013). Test results indicate the likelihood of an individual developing a genetic-related disorder. One of the prime reasons for undertaking genetic testing is to obtain more definite estimates of disease risk (Patenaude, 2005). However, the outcomes of the genetic testing process are often fraught with uncertainty and ambiguity. The extent to which a genetic mutation confers increased disease risk varies markedly across conditions. For some disorders, such as early onset Alzheimer's disease, a genetic mutation will confer close to $100 \%$ likelihood that individuals will develop the condition in their lifetime (Bruni et al., 2014). For many other conditions, however, the degree of disease risk probability is much less (Waalen \& Beutler, 2009), and endogenous causal factors are also implicated (Karageorgos et al., 2015; Lee et al., 2015). For example, one genetic mutation linked with diabetes confers a $16 \%$ risk of developing that disease (Talmud et al., 2010). The existence of multiple causal genes, as is the case for breast and ovarian cancer, makes the interpretation of genetic testing outcomes even more challenging, creating greater ambiguity regarding actual disease risk (Wilson \& Nicholls, 2015). Given that the desire for greater certainty about one's disease susceptibility is a primary motivator for genetic testing (Esplen et al., 2007), the levels of uncertainty implicated with these tests are at the very least challenging and a potential source of frustration and worry. In some cases, genetic testing is not even offered to members of high risk families based on the outcomes of the genetic counseling process, creating yet another source of frustration (Patenaude, 2005).

Information derived from genetic testing can potentially confer numerous benefits, such as informing decision making regarding the long term management of disease risk and motivating preventive actions, particularly if the condition in question is readily treated or prevented. In these cases, genetic-based information potentially empowers individuals and may encourage them to take a more active role in their healthcare decision making. For conditions not currently medically actionable, however, the implications of genetic information are less clear. Genetic testing results are inherently ambiguous, and the complexity and uncertainty entailed in genetic risk information make it challenging for healthcare providers to communicate this information effectively and for tested individuals to comprehend the implications of testing outcomes (Burke \& Korngiebel, 2015; Chowdhury et al., 2015). Individuals using direct-toconsumer testing are particularly vulnerable to misinterpreting genetic risk information due to the minimal clinicbased support provided (Ostergren et al., 2015).

The aim of this special section is to showcase research contributing to our understanding of factors influencing decisions to undergo genetic testing and the impact of the genetic testing process on health-related behaviors of tested individuals. The seven articles fall into three broad groups: (1) identification of factors related to genetic testing uptake and genetic information acceptance; (2) understanding of genetic risk information; and, (3) factors associated with actioning preventive and health-protective behaviors following receipt of genetic risk information. These investigations focus on adult populations within both clinical and community settings, and they address a range of disease types including diabetes, Alzheimer's disease, melanoma, and colorectal, breast and ovarian cancer.

The first two articles report studies investigating factors associated with interest in genetic testing and acceptance of test results (Sherman et al., 2015; Taber et al., 2015b). Using a hypothetical testing scenario in a community sample of adults, Sherman and her colleagues found that interest in clinic-based genetic testing was greater than for direct-to-consumer testing. They also found that interest in testing for Type 2 diabetes overall was greater than for Alzheimer's Disease, the latter condition being perceived as more severe and likely, and less treatable and preventable (i.e., less medically actionable). For direct-toconsumer testing only, interest was greatest for high risk probability (i.e., the degree to which a genetic test result confers increased disease risk). In the second article, Taber and her colleagues used data from a US-adult genome sequencing study to examine the role of perceived ambiguity in genetic testing information regarding decisions to receive testing results. Their findings revealed that perceiving sequencing results as more ambiguous (i.e., as providing less certain information) predicted lower intentions to receive and share testing results; for individuals low in tolerance for uncertainty or optimism, greater perceived ambiguity predicted lower intentions to learn results for non-medically actionable diseases. Both studies highlight the roles of aversion of the ambiguity inherent in 
genetic risk information and the extent to which a disease is perceived as medically actionable as key factors guiding decisions about receiving and communicating genomic information.

The next two articles address the unique contribution of genetic risk information to understanding risk beyond genetic counseling alone (Heiniger et al., 2015; Taber et al., 2015a). Taber, Aspinwall, and their colleagues examined how individuals from melanoma-prone families known to be at risk for a $C D K N 2 A / p 16$ (p16) mutation and who received genetic testing information differed from individuals in melanoma-prone families known to not carry the mutation (who therefore did not undergo genetic testing) in their understanding and acceptance of their melanoma risk status following genetic counseling. Their findings revealed that the individuals receiving genetic test results exhibited less derogation of the accuracy of the risk information, greater understanding of their risk and greater personal applicability of prevention recommendations compared with the individuals ineligible for genetic testing who received counseling alone. Yet despite these benefits of genetic risk information, the research reported by Heiniger and colleagues indicates that individuals are likely to believe this objective risk information is of secondary value in reflecting their disease likelihood and informing their risk management efforts. In a qualitative exploration, they explored women's understanding of breast cancer familial risk for individuals who received genetic testing results and those receiving counseling alone. They found that risk understanding in these women was largely intuitive, an understanding derived from familial and personal experiences with cancer, rather than perceptions shaped by their cognitive understanding of the objective risk information provided by genetic testing. Moreover, these intuitively derived risk perceptions and cognitive risk perceptions shaped by the genetic risk information are held simultaneously. Taken together, the findings from these two studies suggest that whereas objective genetic risk information makes a unique contribution to an individual's understanding of risk, these risk representations are multifaceted and include both cognitive and intuitive perceptions, the latter of which may be strongly influenced by prior experiences within a high risk family.

The final three articles investigate the effects of genetic risk information on beliefs about disease control and prevention (Aspinwall et al., 2015; Kelly et al., 2014; Myers et al., 2015). In a study of individuals receiving testing results for melanoma genetic risk, Aspinwall and her colleagues found that receiving genetic testing information does not generally undermine perceived control, with the majority of participants experiencing either stable or increased perceptions of control over melanoma prevention two years after testing results disclosure. Moreover, increases in perceived control were associated with increases in preventive behaviors, specifically photoprotective clothing use. In a randomized controlled trial, Myers and colleagues investigated the effects of providing genetic and environmental risk information on colorectal cancer screening behaviors. Their findings demonstrate that race is a critical factor in determining subsequent responses to genetic risk information. For individuals at elevated risk, the genetic and environmental risk feedback was found to increase colorectal screening behaviors for white participants, but it had a pronounced negative effect on these behaviors for non-white participants. Finally, the study by Kelly and colleagues reveals that the way in which genetic risk information is conveyed could influence subsequent health protective behaviors. Linguistic analyses of genetic counseling sessions with women undergoing genetic testing for breast and ovarian cancer risk examined the predictive roles of linguistic contents reflecting cognitive processes (e.g., "think", "believe", "understand", etc.) and contents reflecting emotions (e.g., "worried", "relieved", etc.) in cancer screening intentions and behaviors. Both cognitive and affective contents of counseling emerged as key predictors of screening behaviors. These findings highlight the potential importance of tailoring the presentation of genetic counseling information according to both contents reflecting reasoned, conceptual considerations, and contents reflecting emotional experiences and aspects of the testing process.

Taken together, these papers highlight the complexity of genetic testing for disease risk. Clearly the decision to undergo testing in the first instance is not solely a function of membership of a disease-prone family. In particular, they underscore the important roles of multiple cognitive and affective factors in influencing testing and screening decisions. Moreover, the findings from these studies question the implicit assumption that genetic testing feedback will enhance understanding of risk and lead to greater adherence to health-protective actions. Finally, with the growing availability of online, direct-to-consumer genetic testing services, these articles draw attention to the need for well thought-out and tailored counseling approaches, particularly to enhance understanding of genetic risk in members of families ineligible for testing.

This special section provides coverage of a broad range of research questions and contexts, including qualitative and quantitative methodologies; experimental, descriptive, and longitudinal studies; and populations recruited from clinic and community settings. However, the regional settings in which these studies were undertaken are somewhat limited, representing populations drawn from only the United States and Australia. Increasingly, genomics-based technologies are being harnessed in efforts to reduce global health disparities (Bozorgmehr et al., 2011; Williams \& Tishkoff, 2011). Accordingly, behavioral medicine 
research on genomics and genomic testing is expanding to other countries and regions around the globe, including low and low-middle income countries such as Ethiopia (McBride et al., 2015), Tanzania (McBride \& Leppard, 2002), and South Africa (Lund \& Gaigher, 2002). Genomic testing offers new opportunities for health organizations to use genetic information for risk stratification purposes, identifying and targeting scarce resources to those individuals at highest disease risk (McBride et al., 2015). We can expect important cultural differences in reactions to genetic risk information and needs for support and behavior interventions. For example, the findings of Myers and colleagues in particular highlight the ways in which individuals of different races and ethnicities, even those within the same geographical region, can respond differently to genetic risk information. Behavioral medicine research in diverse social and cultural contexts will require cultural expertise, which arguably can be provided most effectively through community-engaged research strategies (Minkler \& Wallerstein, 2011). Behavioral medicine research on genomic testing experiences within diverse cultural communities will critically shape innovations to promote health globally while enriching our theories of risk perception, health behaviors, and health behavior change.

Overall, the papers in this special section of Journal of Behavioral Medicine point to the value of further research to better understand the ways in which individuals perceive, interpret and respond to genetic risk information. With genomics being increasingly integrated into standard medical care, these papers highlight the urgent need to implement evidence-based approaches that address potential inequities in responses to genetic risk in general, and to the way in which genetic risk information is actioned. In particular, we need to minimize potential biases and inequities that may arise due to reluctance to participate in genetic testing due to inherent characteristics such as aversion to ambiguity, and differences in understanding of genetic risk that will impact on subsequent health protective and preventive actions. We need to better understand how to capitalize on factors shown to promote positive health behaviors, whilst acknowledging intrinsic factors and nuances of individual beliefs and experiences that may undermine the genetic risk message. The articles in this special section contribute to this growing body of research, which is critically needed to guide tailored approaches to communicating genetic risk information.

\section{References}

Aspinwall, L. G., Stump, T. K., Taber, J. M., Kohlmann, W., Leaf, S. L., \& Leachman, S. A. (2015). Impact of melanoma genetic test reporting on perceived control over melanoma prevention. Journal of Behavioral Medicine. doi:10.1007/s10865-0159631-8.

Barlow-Stewart, K., \& Parasivam, G. (2007). DNA genetic testingscreening for genetic conditions and genetic susceptibility [Fact sheet]. In K. Barlow-Stewart, G. Parasivam \& Centre for Genetics Education (N.S.W.) (Eds.), The Australasian genetics resource book. St Leonards, N.S.W.: Centre for Genetics Education.

Beachy, S., Olson, S., \& Berger, A. (2015). Genomics-enabled learning health care systems: Gathering and using genomic information to improve patient care and research: Workshop summary. Washington, DC: The National Academies Press.

Bozorgmehr, K., Saint, V. A., \& Tinnemann, P. (2011). The 'global health' education framework: A conceptual guide for monitoring, evaluation and practice. Global Health, 7, 8. doi:10.1186/ 1744-8603-7-8

Bruni, A. C., Conidi, M. E., \& Bernardi, L. (2014). Genetics in degenerative dementia: Current status and applicability. Alzheimer's Disease and Associated Disorders, 28, 199-205. doi:10. 1097/WAD.0000000000000046

Burke, W., \& Korngiebel, D. M. (2015). Closing the gap between knowledge and clinical application: Challenges for genomic translation. PLoS Genetics, 11, e1004978. doi:10.1371/journal. pgen. 1004978

Caulfield, T., Ries, N. M., Ray, P. N., Shuman, C., \& Wilson, B. (2010). Direct-to-consumer genetic testing: Good, bad or benign? Clinical Genetics, 77, 101-105. doi:10.1111/j.13990004.2009.01291.x

Chowdhury, S., Henneman, L., Dent, T., Hall, A., Burton, A., Pharoah, P., \& Burton, H. (2015). Do health professionals need additional competencies for stratified cancer prevention based on genetic risk profiling? Journal of Personalized Medicine, 5, 191-212. doi:10.3390/jpm5020191

Esplen, M. J., Madlensky, L., Aronson, M., Rothenmund, H., Gallinger, S., Butler, K., \& McLaughlin, J. (2007). Colorectal cancer survivors undergoing genetic testing for hereditary nonpolyposis colorectal cancer: Motivational factors and psychosocial functioning. Clinical Genetics, 72, 394-401. doi:10.1111/j. 1399-0004.2007.00893.x

Heiniger, L., Butow, P. N., Charles, M., kConFab Psychosocial Group on behalf of the kConFab Investigators., \& Price, M. A. (2015). Intuition versus cognition: A qualitative exploration of how women understand and manage their increased breast cancer risk. Journal of Behavioral Medicine. doi:10.1007/s10865-0159632-7.

Karageorgos, I., Mizzi, C., Giannopoulou, E., Pavlidis, C., Peters, B. A., Zagoriti, Z., \& Patrinos, G. P. (2015). Identification of cancer predisposition variants in apparently healthy individuals using a next-generation sequencing-based family genomics approach. Human Genomics, 9, 12. doi:10.1186/s40246-015-0034-2

Kelly, K. M., Ellington, L., Schoenberg, N., Jackson, T., Dickinson, S., Porter, K., Leventhal, H., \& Andrykowski, M. (2014). Genetic counseling content: How does it impact health behavior? Journal of Behavioral Medicine. doi:10.1007/s10865-014-96132.

Lee, J. U., Kim, J. D., \& Park, C. S. (2015). Gene-environment interactions in asthma: Genetic and epigenetic effects. Yonsei Medical Journal, 56, 877-886. doi:10.3349/ymj.2015.56.4.877

Lund, P. M., \& Gaigher, R. (2002). A health intervention programme for children with albinism at a special school in South Africa. Health Education and Research, 17, 365-372.

Myers, R. E., Ruth, K., Manne, S. L., Cocroft, J., Sifri, R., Ziring, B., Burgh, D., Ross, E., \& Weinberg, D. S. (2015). Effects of genetic and environmental risk assessment feedback on colorectal cancer 
screening adherence. Journal of Behavioral Medicine. doi:10. 1007/s10865-015-9626-5.

McBride, C. M., Birmingham, W. C., \& Kinney, A. Y. (2015). Health psychology and translational genomic research: Bringing innovation to cancer-related behavioral interventions. American Psychologist, 70, 91-104. doi:10.1037/a0036568

McBride, S. R., \& Leppard, B. J. (2002). Attitudes and beliefs of an albino population toward sun avoidance: Advice and services provided by an outreach albino clinic in Tanzania. Archives of Dermatology, 138, 629-632.

Minkler, M., \& Wallerstein, N. (2011). Community-based participatory research for health: From process to outcomes. San Francisco, CA: Jossey-Bass.

National Health and Medical Research Council. (2012). Direct-toconsumer DNA genetic testing: An information resource for consumers. Retrieved from https://www.nhmrc.gov.au/guide lines-publications/ps4

National Institutes of Health. (2013). Confused by genetic tests? NIH's new online tool may help. http://www.nih.gov/news/ health/feb2012/od-29.htm

Ostergren, J. E., Gornick, M. C., Carere, D. A., Kalia, S. S., Uhlmann, W. R., Ruffin, M. T., \& Roberts, J. S. (2015). How well do customers of direct-to-consumer personal genomic testing services comprehend genetic test results? Findings from the Impact of Personal Genomics Study. Public Health Genomics, 1, 182-200. doi:10.1159/000431250

Patenaude, A. F. (2005). Genetic testing for cancer: Psychological approaches for helping patients and families. Washington, DC: American Psychological Association.

Sherman, K., Shaw, L.-K., Champion, K., Caldeira, F., \& McCaskill, M. (2015). The effect of disease risk probability and disease type on interest in clinic-based versus direct-to-consumer genetic testing services. Journal of Behavioral Medicine. doi:10.1007/ s10865-015-9630-9.

Taber, J. M., Aspinwall, L. G., Stump, T. K., Kohlmann, W., Champine, M., \& Leachman S. A. (2015a). Genetic test reporting enhances understanding of risk information and acceptance of prevention recommendations compared to family history-based counseling alone. Journal of Behavioral Medicine. doi:10.1007/s10865-015-9648-z.

Taber, J. M., Klein, W. M. P., Ferrer, R. A., Han, P. K. J., Lewis, K. L., Biesecker, L. G., \& Biesecker, B. B. (2015b). Perceived ambiguity as a barrier to intentions to learn genome sequencing results. Journal of Behavioral Medicine. doi:10.1007/s10865015-9642-5.

Talmud, P., Hingorani, A., Cooper, J., Marmot, M., Brunner, E., Kumari, M., \& Humphries, S. (2010). Utility of genetic and nongenetic risk factors in prediction of type 2 diabetes: Whitehall II prospective cohort study. The British Medical Journal, 340, $1-10$.

Waalen, J., \& Beutler, E. (2009). Genetic screening for lowpenetrance variants in protein-coding genes. Annual Review of Genomics and Human Genetics, 10, 431-450. doi:10.1146/ annurev.genom.9.081307.164255

Williams, S., \& Javitt, G. (2008). Direct-to-consumer genetic testing: Empowering or endangering the public? [Issue brief]. Washington, DC: Genetics and Public Policy Center.

Williams, S. M., \& Tishkoff, S. A. (2011). Exploring genomic studies in Africa. Genome Medicine, 3, 45. doi:10.1186/gm261

Wilson, B. J., \& Nicholls, S. G. (2015). The Human Genome Project, and recent advances in personalized genomics. Risk Management and Healthcare Policy, 8, 9-20. doi:10.2147/RMHP. S58728 